\title{
Formation of dodecagonal quasicrystals in two-dimensional systems of patchy particles
}

\author{
Marjolein N. van der Linden, ${ }^{1, a)}$ Jonathan P. K. Doye,, b) and Ard A. Louis ${ }^{2}$ \\ 1) Physical and Theoretical Chemistry Laboratory, Department of Chemistry, University of Oxford, South Parks Road, \\ Oxford, OX1 3QZ, United Kingdom \\ ${ }^{2)}$ Rudolf Peierls Centre for Theoretical Physics, University of Oxford, 1 Keble Road, Oxford, OX1 3NP, \\ United Kingdom
}

(Dated: 15 October 2018)

The behaviour of two-dimensional patchy particles with 5 and 7 regularly-arranged patches is investigated by computer simulation. For higher pressures and wider patch widths, hexagonal crystals have the lowest enthalpy, whereas at lower pressures and for narrower patches, lower-density crystals with five nearest neighbours and that are based on the $\left(3^{2}, 4,3,4\right)$ tiling of squares and triangles become lower in enthalpy. Interestingly, in regions of parameter space near to that where the hexagonal crystals become stable, quasicrystalline structures with dodecagonal symmetry form on cooling from high temperature. These quasicrystals can be considered as tilings of squares and triangles, and are probably stabilized by the large configurational entropy associated with all the different possible such tilings. The potential for experimentally realizing such structures using DNA multi-arm motifs are discussed.

PACS numbers: 61.44.Bri,47.57.-s,81.16.Dn

\section{INTRODUCTION}

Since the formation of quasicrystals were first reported in 1984 by Shechtman et al. for an Al-Mn alloy, many systems have been to found to exhibit a quasicrystalline phase. Most of these are binary or ternary metallic alloys (but never a pure metal). The quest to find quasicrystals beyond alloys has led to an increasing number of examples, mostly in the field of soft condensed matter.$^{2]}$ Examples include dendrimers, $\frac{314}{4}$ star polymers, ${ }^{[5}$ micelles ${ }^{6}$ and binary mixtures of nanoparticles. ${ }^{7}$ All of these examples, except for one of the micellar systems, ${ }^{6}$ have dodecagonal symmetry and are often found in regions of parameter space close to where crystalline approximants, such as the Frank-Kasper $\sigma$ phase, are observed 13

A remaining target is to find a colloidal system that can self-assemble into a quasicrystalline structure in the absence of an external field (two-dimensional colloidal quasicrystals can be induced to form using quasiperiodic light fields $14-16)$. One approach might be to use a binary or ternary colloidal mixture, but although complex crystal structures have been reported for binary mixtures, 17 21 as yet no quasicrystals have been observed. Another approach might be to use colloids with anisotropic "patchy" interactions, 22 where the positions of the patches could be used to control the preferred local geometry and hence influence the global structure formed. Indeed, much progress has been made in developing methods to synthesize such types of colloidal particles. ${ }^{23}$ Furthermore, the first experiments on the

\footnotetext{
a) Present address: Soft Condensed Matter, Debye Institute for Nanomaterials Science, Utrecht University, Princetonplein 5, 3584 CC Utrecht, The Netherlands

b) Author for correspondence
}

novel structures that such patchy interactions can enable the systems to adopt are beginning to appear, 33 as well as being systematically explored through computer simulations. $\underline{34}$

Quasicrystals have also been found to form for a variety of model potentials in computer simulations. Interestingly, these are not restricted to mixtures, ${ }^{44 \mid 45}$ but can also occur for one-component systems. $\stackrel{467}{5 x}$ Examples include isotropic pair potentials with both a maximum and

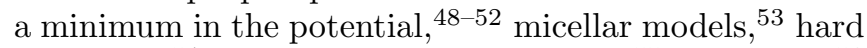
tetrahedra ${ }^{54}$ and triangular bipyramids, $\frac{55}{5}$ and water ${ }^{56}$ and silicon $\stackrel{57}{57}$ bilayers.

Here, we wish to examine in detail the behaviour of two-dimensional patchy particles with 5 and 7 patches as possible quasicrystal-forming systems. The reason we choose this system is that in a recent study of twodimensional disks with regularly arranged patches, intriguing behaviour was seen for the 5-patch system. Hard disks naturally form a hexagonal crystal, and six patches reinforce this tendency. For four patches, there is a competition between a square crystal, which is energetically stabilized by the patchy interactions, and a hexagonal crystal, which is stabilized by its higher density. However, the situation for particles with 5 regularly arranged patches is more complex, since the 5 -fold symmetry of the particles is incompatible with crystalline order - there is no crystal in which all the patches can point directly at those on neighbouring particles.

In Ref. 35 we found that on cooling (at low pressure), crystallization was not observed, because of the frustration introduced by the geometry of the patches. The configurations generated did though show certain common local motifs (those shown in Fig. 1) and could be considered as tilings of squares and triangles. Although there was no overall crystalline order, there was some evidence suggestive of longer-range orientational order; how- 

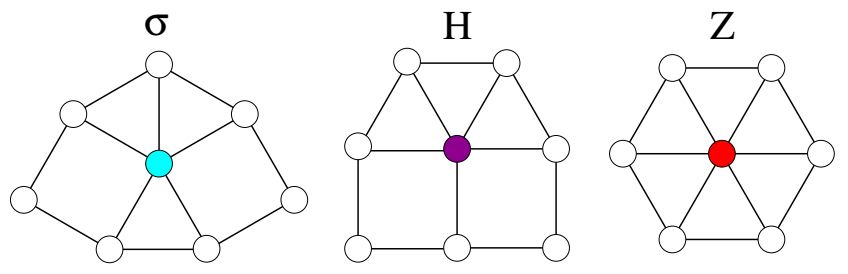

FIG. 1. The three local environments that are mainly present in the simulated structures.

ever, because of the relatively small systems sizes considered this was not pursued further at the time. Given the known tendency of random square-triangle tilings to form dodecagonal quasicrystals, $\frac{58}{5}$ we now investigate in detail the suggestion made in Ref. 35 that this system may form quasicrystals.

\section{METHODS}

\section{A. Potential}

The model consists of spherical particles patterned with attractive patches. They are described by a modified Lennard-Jones potential, in which the repulsive part of the potential is isotropic, but the attractive part is anisotropic and depends on the alignment of patches on interacting particles. Specifically, the potential is described by

$$
V_{i j}\left(\mathbf{r}_{i j}, \boldsymbol{\Omega}_{i}, \boldsymbol{\Omega}_{j}\right)= \begin{cases}V_{\mathrm{LJ}}\left(r_{i j}\right) & r<\sigma_{\mathrm{LJ}} \\ V_{\mathrm{LJ}}\left(r_{i j}\right) V_{\mathrm{ang}}\left(\hat{\mathbf{r}}_{i j}, \boldsymbol{\Omega}_{i}, \boldsymbol{\Omega}_{j}\right) & r \geq \sigma_{\mathrm{LJ}}\end{cases}
$$

where $V_{\mathrm{LJ}}$, the Lennard-Jones potential, is given by

$$
V_{\mathrm{LJ}}(r)=4 \epsilon\left[\left(\frac{\sigma_{\mathrm{LJ}}}{r}\right)^{12}-\left(\frac{\sigma_{\mathrm{LJ}}}{r}\right)^{6}\right] .
$$

The minimum of this potential is at $r_{\mathrm{eq}}=2^{1 / 6} \sigma_{\mathrm{LJ}} . V_{\text {ang }}$ is an angular modulation factor that depends on the orientations of the patches on the two interacting particles with respect to the interparticle vector. Specifically,

$$
V_{\text {ang }}\left(\hat{\mathbf{r}}_{i j}, \boldsymbol{\Omega}_{i}, \boldsymbol{\Omega}_{j}\right)=G_{i j}\left(\hat{\mathbf{r}}_{i j}, \boldsymbol{\Omega}_{i}\right) G_{j i}\left(\hat{\mathbf{r}}_{j i}, \boldsymbol{\Omega}_{j}\right),
$$

where

$$
G_{i j}\left(\hat{\mathbf{r}}_{i j}, \boldsymbol{\Omega}_{i}\right)=\exp \left(-\frac{\theta_{k_{\min } i j}^{2}}{2 \sigma_{\mathrm{pw}}^{2}}\right)
$$

$\sigma_{\mathrm{pw}}$ is a measure of the angular width of the patches, $\theta_{k i j}$ is the angle between patch vector $k$ on particle $i$ and the interparticle vector $\mathbf{r}_{i j}$, and $k_{\min }$ is the patch that minimizes the magnitude of this angle. Hence, only the patches on each particle that are closest to the interparticle vector interact with each other, and $V_{\text {ang }}=1$ if the patches point directly at each other. One feature of this potential is that as $\sigma_{\mathrm{pw}} \rightarrow \infty$ the isotropic Lennard-Jones potential is recovered. For computational efficiency the potential is truncated and shifted at $r=3 \sigma_{\mathrm{LJ}}$, and the crossover distance in Eq. 1 is adjusted so that it still occurs when the potential is zero.

As well as studying crystalization in two

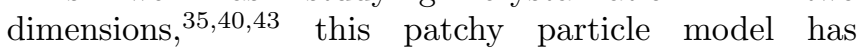
also been previously used to study crystallization in three dimensions, $35 \sqrt{37}$ and the self-assembly of monodisperse shells. ${ }^{[9]}[1]$

\section{B. Simulation}

Systems of particles were simulated using standard Metropolis Monte Carlo (MC) in the NPT ensemble. Periodic boundary conditions were applied, and the simulation box was constrained to be square. The MC moves were single-particle rotational and translational moves, as well as "volume" moves in which the area of the box was scaled, the latter allowing a constant pressure ensemble to be simulated. The number of particles $N$ was always 2500 .

\section{Structural analysis}

We employ a number of approaches to analyse the structures that the systems of patchy particles adopt. In the condensed state, we find that the 5- and 7-patch particles virtually always adopt one of three local environments. These are illustrated in Fig. 1. There are two possible five-coordinate environments, which are based upon two different ways of locally packing squares and triangles. We refer to them as the $\sigma$ and $H$ environments by analogy to the Frank-Kasper phases of these names. ${ }^{62 / 63}$ These $\sigma$ and $H$ Frank Kasper phases are crystalline approximants to dodecagonal quasicrystals and can be considered as square-triangle tilings in two of their three dimensions ${ }^{64}$ The tilings containing only these local environments are more formally denoted by $\left(3^{2}, 4,3,4\right)(\sigma)$ and $\left(3^{3}, 4^{2}\right)(H)$, where this nomenclature refers to the sequence of polygons around a vertex $\frac{65}{65}$ The third local environment is the six-coordinate hexagonal environment, which represents the densest local packing, and will be denoted $Z$, again by analogy to a Frank-Kasper phase.

To identify these environments we employ a common neighbour analysis. Neighbours are defined as all particles within a certain cutoff radius $r_{c}$ from a given particle. Then for each pair of neighbours the number of neighbours common to both is determined. Each local environment is characterized by a unique signature in terms of the number of neighbours with which the central particle shares a certain number of neighbours. For example, a particle in a $\sigma$ local environment has a total of five neighbours, with one of which it has two neighbours in common and with four of which it has one neighbour in common. Hence, this environment is denoted by the 
common neighbour signature $\{21111\}$. In a similar way, the signatures for the $H$ and $Z$ local environments are $\{22110\}$ and $\{222222\}$, respectively. Particles that are not in any of these three local environments are labelled 'undefined' $(U)$. The cutoff radius $\left(r_{c} \approx 1.38 \sigma_{\mathrm{LJ}}\right)$ was chosen such that the fraction of $U$-particles was minimized.

To probe the global order of the configurations generated, the associated diffraction patterns were calculated. Quasicrystalline configurations are characterized by diffraction patterns with non-crystallographic rotational symmetries. We calculated the diffraction patterns by evaluating the real part of the interference function:

$$
S(\mathbf{q})=\frac{1}{N} \sum_{i=0}^{N-1} \sum_{j=0}^{N-1} \exp \left[2 \pi \mathrm{iq} \cdot\left(\mathbf{r}_{i}-\mathbf{r}_{j}\right)\right]
$$

where $\mathbf{q}$ is the wavevector and $\mathbf{r}_{i}$ the position of particle $i$. Unless stated otherwise, the resolution we used for the plots of the diffraction patterns is $\Delta q_{x}=\Delta q_{y}=0.15 \sigma_{\mathrm{LJ}}^{-1}$.

When mapping out the behaviour of our system as a function of the parameter space it will be useful to to measure the degree of twelvefold symmetry of the diffraction pattern. To achieve this, we take a Fourier transform around a ring of the interference pattern at a $q$ value corresponding to the first diffraction peaks. Specifically, we evaluate

$$
F(\nu)=\sum_{i=0}^{n-1} S\left(q_{1 \mathrm{st}}, \phi_{i}\right) \exp \left(-2 \pi \mathrm{i} \nu \phi_{i}\right),
$$

where the sum is over the $n$ sampled points of $S\left(q_{1 \mathrm{st}}, \phi\right)$. A twelvefold symmetric diffraction pattern will have high values at $\nu=12$ and multiples thereof. Similarly, a sixfold symmetric diffraction pattern, as would be expected for a hexagonal crystal, will have high values at $\nu=6$ and multiples thereof (including $\nu=12$ ). Therefore, we used $|F(\nu=12)|-|F(\nu=6)|$ as a measure of the twelvefold character of the diffraction pattern. We note that the peaks at multiples of the lowest frequency will have a lower amplitude than the main lowest frequency peak, where this decay in amplitude is stronger if the diffraction peaks are more diffuse.

\section{RESULTS}

We will first consider the 5-patch particles in detail. The behaviour of the 7-patch particles is fairly similar and so we will consider this system more briefly in Section IIIB.

\section{A. 5-patch particles}

\section{Low-enthalpy structures}

The most stable phase at zero temperature is simply that with the lowest enthalpy. Therefore, we minimized

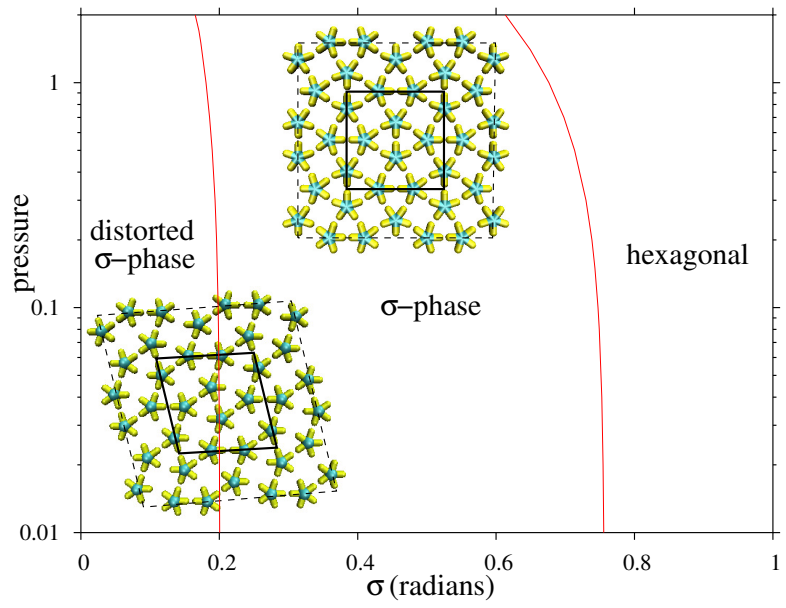

FIG. 2. Zero-temperature phase diagram showing the dependence of the lowest-enthalpy structure on pressure and patch width.

the enthalpy for a variety of candidate crystal structures under different conditions and potential parameters in order to obtain Figure 2, which shows how the lowest enthalpy crystal structure depends on pressure and patch width. This figure is slightly different from that which appeared in Ref. 35, because a slightly lower energy crystal at low $\sigma_{\mathrm{pw}}$ was subsequently identified in Ref. 40

At sufficiently high pressure the most stable phase will be the crystal with highest density, which in this system is the hexagonal crystal. At sufficiently low pressure, the crystal structure with the lowest energy will be most stable, and will be the one which maximizes the patchpatch interactions.

At intermediate values of the patch width, the low energy crystals are those based on the two five-coordinate local environments depicted in Fig. 1. Although the patches are not able to point directly at those on neighbouring particles in these motifs, the loss in energy is not prohibitive because the angular deviations are relatively small (Fig. 3). As the average angular deviation is smaller in the $\sigma$ local environment, the $\sigma$ crystal is slightly lower in energy than the $H$ crystal.

As the patches becomes narrower, the energetic penalty associated with the non-perfect alignment of the patches with the interparticle vectors increases, until a point is reached where it becomes favourable for the local environments to distort so that the patches point directly at three of the five neighbours. The resulting packings can be considered to be made of the irregular hexagon shown in Fig. 3(c). For the $H$ crystal this distortion does not lead to any change in its space group, namely it remains as $\mathrm{cmm}$. However, for the $\sigma$ crystal there is a symmetry breaking - there are two equivalent ways of dividing up the structure into these hexagons - and the space group changes from $p 4 g$ to $p 2$. The resulting crystals are virtually degenerate, with the distorted $\sigma$ structure just lower in energy 40

As the patch width is increased away from the inter- 

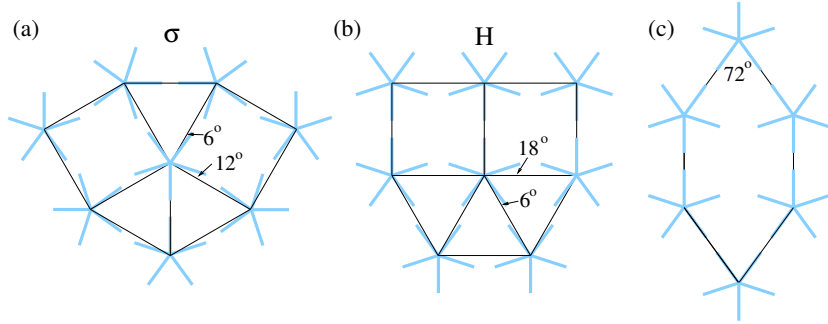

FIG. 3. Deviations of the patch vectors from the interparticle vectors in the (a) $\sigma$ - and (b) $H$-type environments. (c) At low values of the patch width it is favourable for these structures to distort so that three of the patches point directly at the neighbouring particles. The resulting structures can be viewed as being made up of the depicted hexagon.

mediate values at which the $\sigma$ phase is stable, the orientational dependence of the potential becomes weaker, and there comes a point at which the hexagonal crystal becomes lowest in energy because of its greater number of neighbours.

In the $\sigma$-phase the ratio of triangles to squares is 2 . There are also many larger-unit cell crystal structures with a mixture of $\sigma$ and $Z$ environments that have an increasing ratio of triangles to squares. We thought that these more complex crystals might be most stable near to the $\sigma$ /hexagonal boundary in Fig. 2. However, we never found an instance where one of these structures had the lowest enthalpy.

\section{Annealing}

We do not expect quasicrystalline configurations to be lowest in enthalpy, both because the closest quasicrystalline approximants (the crystals involving both $\sigma$ and $Z$ local environments) are never lowest in enthalpy (Sect. III A 1 and because the disorder associated with quasicrystals is likely to lead them to have a somewhat higher enthalpy than the relevant approximant. However, it may be that a quasicrystal is more kinetically accessible than possible crystals on cooling, or is even thermodynamically stable for a particular temperature range due to its greater entropy. Therefore, to search for quasicrystalline behaviour we performed a series of cooling runs for a grid of pressure and patch width values. In these runs the temperature was decreased linearly from $0.5 \epsilon k^{-1}$ to zero over $50000 \mathrm{MC}$ cycles. Some of the resulting configurations did show twelve-fold diffraction patterns characteristic of a dodecagonal quasicrystal. However, the peaks were very diffuse. Therefore, we subsequently annealed all the final configurations at a temperature of $0.15 \epsilon k^{-1}$ for $10^{6} \mathrm{MC}$ cycles. This temperature was chosen so that the mobility of the atoms was large enough to allow significant ordering during annealing. For the most part, this temperature was also below that at which the quasicrystals formed, the exceptions occurring near to
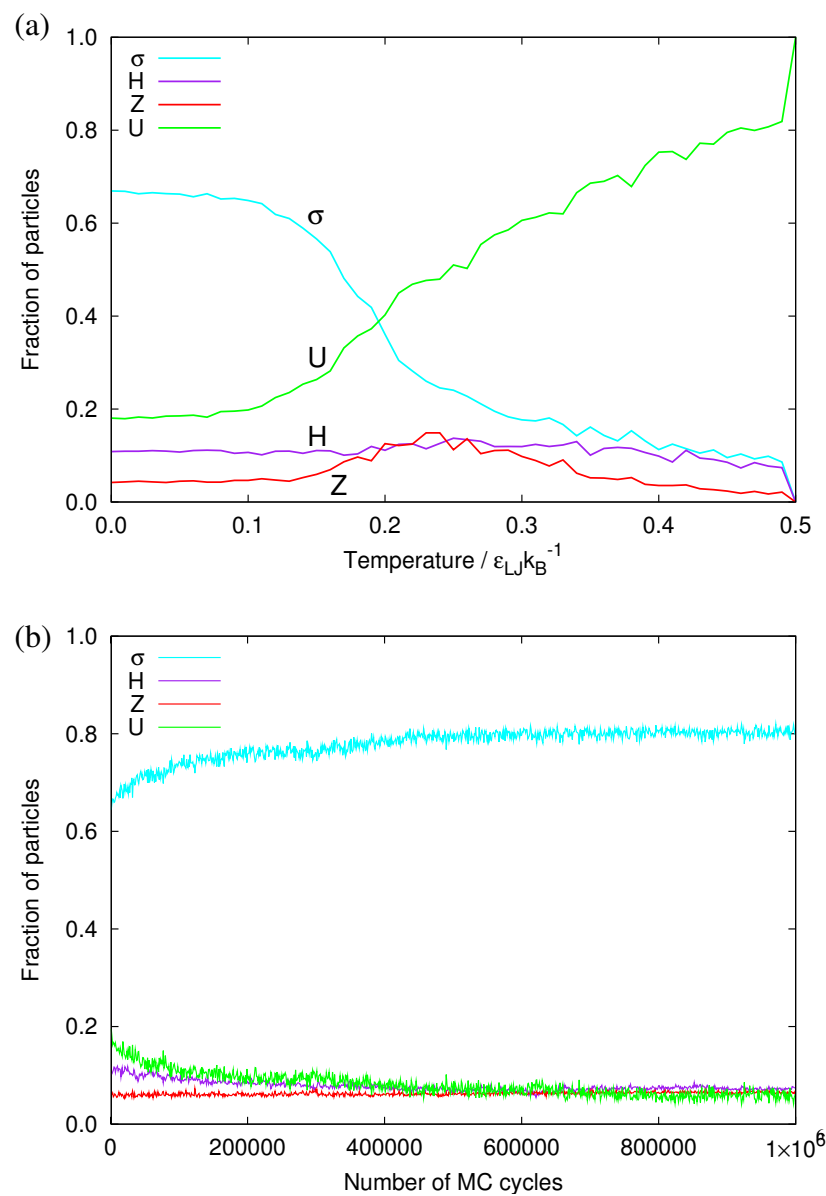

FIG. 4. Evolution of local structural environments during (a) cooling and (b) annealing at $T=0.15 \epsilon k^{-1} \cdot p=0.7 \epsilon \sigma_{\mathrm{LJ}}^{-2}$, $\sigma_{\mathrm{pw}}=0.49$.

the hexagonal $/ \sigma$ boundary in Fig. 2 , where a hexagonal phase formed first on cooling before transforming into the quasicrystalline phase at lower temperature.

Figure 4 shows the evolution of the number of particles with different local environments for the cooling and annealing runs for a state point that led to a dodecagonal quasicrystal. On cooling, the number of $\sigma$ environments increases rapidly between 0.2 and $0.1 \epsilon k^{-1}$ and is due to the formation of the quasicrystal. Prior to this there is a transient increase in the number of hexagonal particles. Closer to the hexagonal $/ \sigma$ boundary in Fig. 2 , this transient increase is more pronounced because of the increased stability of the hexagonal phase. Even though the hexagonal crystal is not the lowest enthalpy structure under these conditions, it is thermally stabilized by its large orientational entropy.

It is noteworthy that the number of hexagonal particles does not decrease to zero on quasicrystal formation. This is not due to incomplete ordering but is one of the features of the quasicrystalline structures. At the end of the cooling run, there is still a significant fraction of particles whose local structure cannot be assigned because of the disorder within the configurations. 
(a)

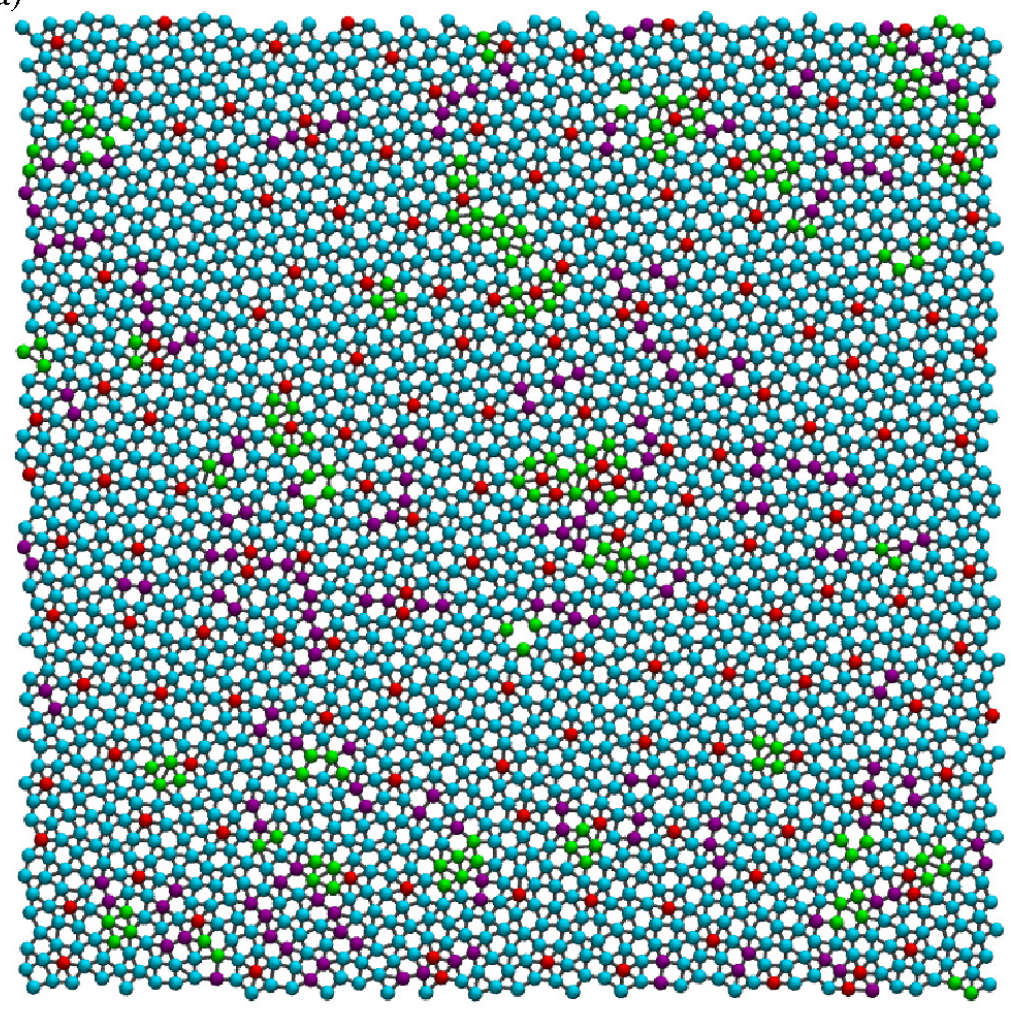

(b)

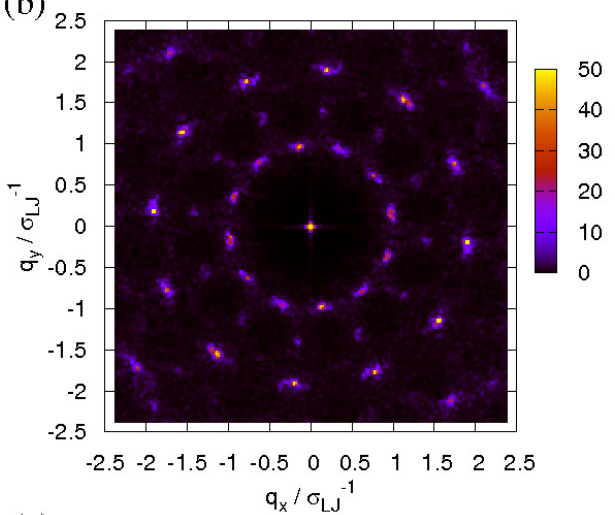

(c)

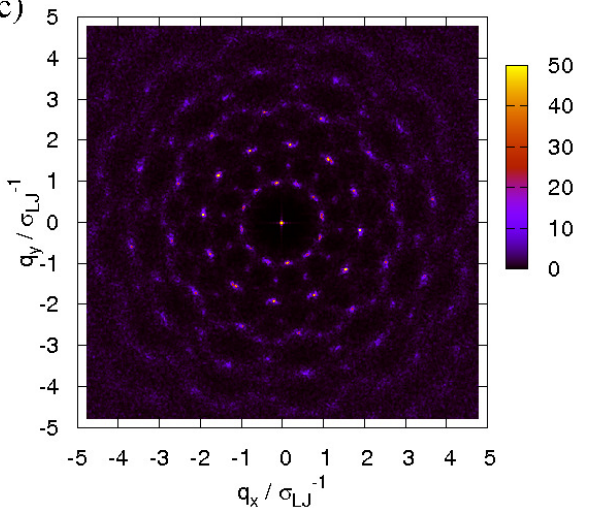

FIG. 5. (a) Configuration after annealing and (b) and (c) associated diffraction patterns (for two different ranges of $\mathbf{q}$ ) at $p=0.7 \epsilon \sigma_{\mathrm{LJ}}^{-2}, \sigma_{\mathrm{pw}}=0.49$.

On annealing the number of $\sigma$ environments gradually increases at the expense of unidentified and $H$ environments (but not hexagonal) (Fig. 4(b)). The resulting configuration is shown in Fig. 5 along with the associated diffraction pattern. The diffraction pattern shows clear twelvefold symmetry and is very similar to that for a "perfect" dodecagonal quasicrystal produced by the "extended Schlottmann" inflation rules ${ }^{66}$ (Supplementary Fig. 1), albeit with less sharp peaks. The twelvefold pattern also implies that the orientational order is coherent across the whole box; it could be said to be a "single quasicrystal".

The configuration is a square-triangle tiling in which the twelve possible orientations of the bond vectors are equally likely - hence, the twelvefold symmetry in the diffraction pattern. The radial distribution function shows clear peaks out to quite long range (Fig. 6(a)) because of the square-triangle order. Close inspection of the configuration shows that the hexagonal atoms for the most part do not cluster together but are instead isolated from each other and are usually at the centre of the dodecagonal motifs depicted in Fig. 7(a) and (b). These dodecagonal motifs can join together in two ways. They can share an edge as in Fig. 7(c) (their centres are separated by $\left.(2+\sqrt{3}) r_{\text {eq }}=4.189 \sigma_{\mathrm{LJ}}\right)$ or interpenetrate as in Fig. 7(e) (separation $\left.(1+\sqrt{3}) r_{\mathrm{eq}}=3.067 \sigma_{\mathrm{LJ}}\right)$. These two distances are very apparent from the radial distri- bution function for particles in hexagonal environments (Fig. 6(b)) with a clear preference for edge-sharing dodecagons.

The four basic ways of locally packing the dodecagons that are possible using interpenetrating and edge-sharing dodecagons are illustrated in Fig. 7(c)-(f), two of which are triangular, one of which is square and one of which is rectangular. These motifs can be used to construct a whole variety of crystal structures with large unit cells (and varying ratios of squares to triangles), although as mentioned in Sect. IIIA1 we did not find an instance where these crystals had the lowest enthalpy. The model quasicrystal that we created using the extended Schlottmann inflation rules (Supplementary Fig. 1) can also be analysed in terms of the edge-sharing dodecagon motifs of Fig. 7( (c) and (d).

When the configuration in Fig. 5(a) is examined all four of these motifs can be found, with the triangle of edge-sharing dodecagons (Fig. 77(c)) most common. When examining how these triangular, square and rectangular elements begin to tile the plane, we find configurations like those in Fig. 1. but now, of course, on a longer length scale. Furthermore, this tiling seems to be random and does not completely tile the plane, because of a significant fraction of defects, both in terms of $U$ particles with an "unidentified" coordination shell and strings of $H$ particles. It is noteworthy that $\sigma$ and $H$ 

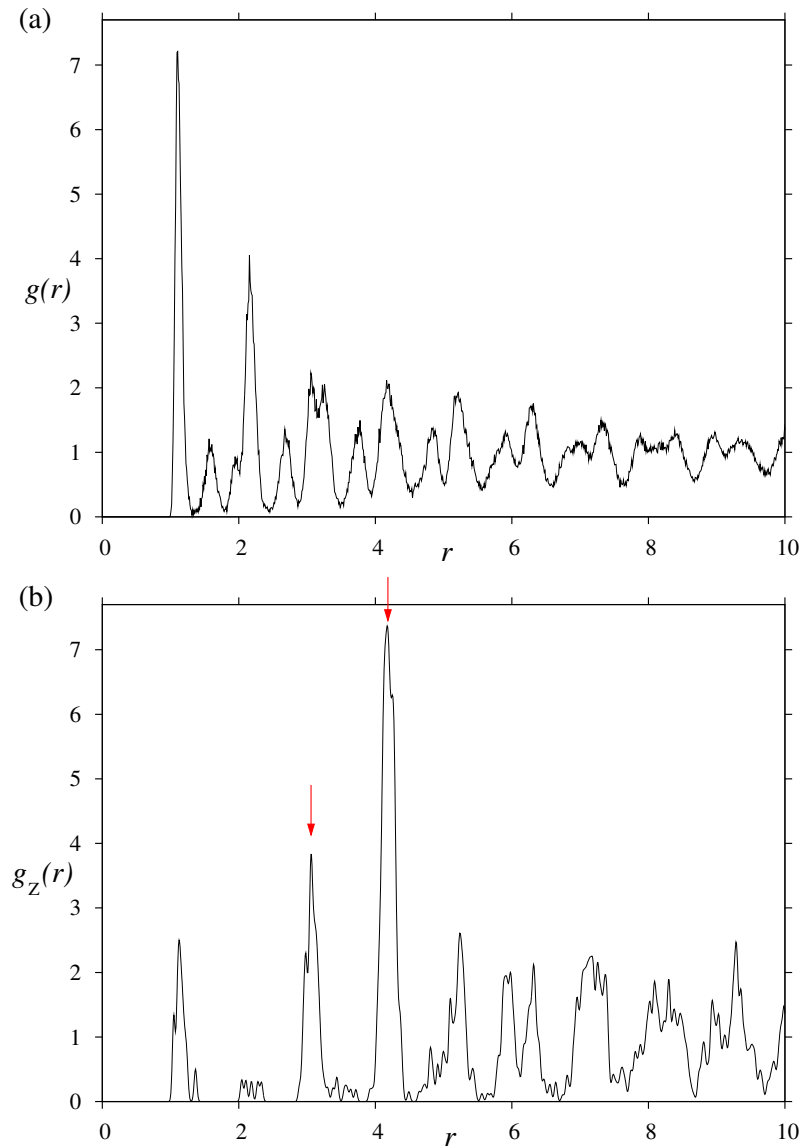

FIG. 6. Radial distribution functions for (a) all the particles and (b) the $Z$ particles in the quasicrystalline configuration obtained at $p=0.7 \epsilon \sigma_{\mathrm{LJ}}^{-2}, \sigma_{\mathrm{pw}}=0.49$. In (b) the peaks associated with interpenetrating (Fig. 7(e)) and edge-sharing (Fig. 7.(c)) dodecagons are marked with arrows.
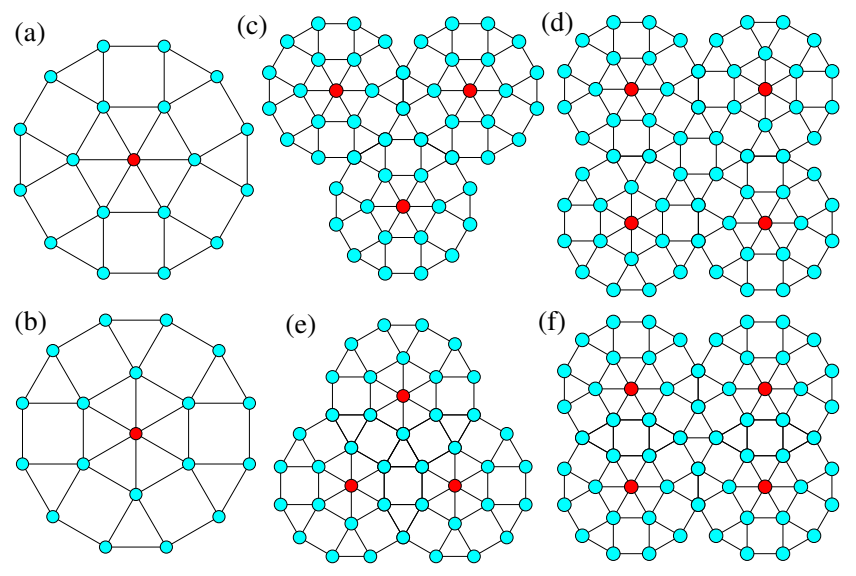

FIG. 7. (a) and (b) Dodecagonal motifs, and (c)-(f) four ways of locally packing these motifs. crystals can form a coherent interface along the $\{11\}$ directions of the $\sigma$ crystals, and the latter defects seem to often occur when the $\sigma$-like order in two adjacent regions is not fully in registry, and needs a line of $H$ atoms to bridge the regions.

Another source of disorder is the two possible orientations of the central hexagon in the dodecagonal motif, as illustrated in Fig. 7(a) and (b). The two forms can be transformed into each other by a rotation of the hexagon by $\pi / 6$. For the edge-sharing dodecagons in Fig. 7 the orientations of the hexagons have been chosen so that all the particles on the edge of the dodecagons have the more favourable $\sigma$ environments. However, some relative orientations of the internal hexagons lead to $H$ environments. For example, there are two $H$ environments at the shared edge if both hexagons have bonds parallel to the shared edge, and a few examples of such $H$ "dimers" can be found in Fig. 5(a). By contrast, for the interpenetrating dodecagons (Fig. 7(d) and (e)), if the hexagon at the centre of one of the dodecagons is rotated, the dodecagonal character of the other centres is lost, although the packing is still a square-triangle tiling.

Another way to characterize the structures that we observe is in terms of the ratio of triangles to squares in the tiling, and the related ratio of the number of five- and six-coordinate atoms. For example, we find the ratio of triangles to squares for the configuration in Fig. 5(a) to be 2.303, which compares with a value of 2 for the $\sigma$ crystal and $4 / \sqrt{3}=2.309$ for an "extended Schlottmann" quasicrystal ${ }^{69}$ Similarly, the ratio of 5- to 6-coordinate environments is 13.194 for the configuration in Fig. 5(a), which is again similar to the value for an "extended Schlottmann" quasicrystal, namely $(24+14 \sqrt{3}) /(2+\sqrt{3})=12.928$. These results clearly indicate the close similarity of the structures we obtain to ideal dodecagonal quasicrystals.

So far we have only looked at the structure that results for one particular set of conditions. Figure 8 provides an overview of the structural behaviour as a function of pressure and patch width, and Figure 9 provides example final configurations for different patch widths at a representative pressure.

In the top right corner of the $\left(\sigma_{\mathrm{pw}}, P\right)$ plane, i.e. larger patch widths and higher pressures, the hexagonal phase is most stable (Figure 2). As one moves away from this corner of the parameter space to lower pressures and patch widths, the onset of quasicrystal formation is signalled by a sharp increase in the number of $\sigma$ environments at the expense of hexagonal environments (Fig. 8(a) and (b)). Close to this boundary, our measure of the twelvefoldness of the diffraction pattern generally has high values, although this measure should be interpreted with caution as false positives can arise. For example, the superposition of diffraction patterns from two different hexagonal domains leads to an anomalously high value of the twelvefoldness at $P=1.7 \epsilon \sigma_{\mathrm{LJ}}^{-2}$ and $\sigma_{\mathrm{pw}}=0.63$. The position of the hexagonal to quasicrystal boundary in Fig. 8 is noticeably to the left of the zero-temperature $\sigma$-hexagonal 
(a)
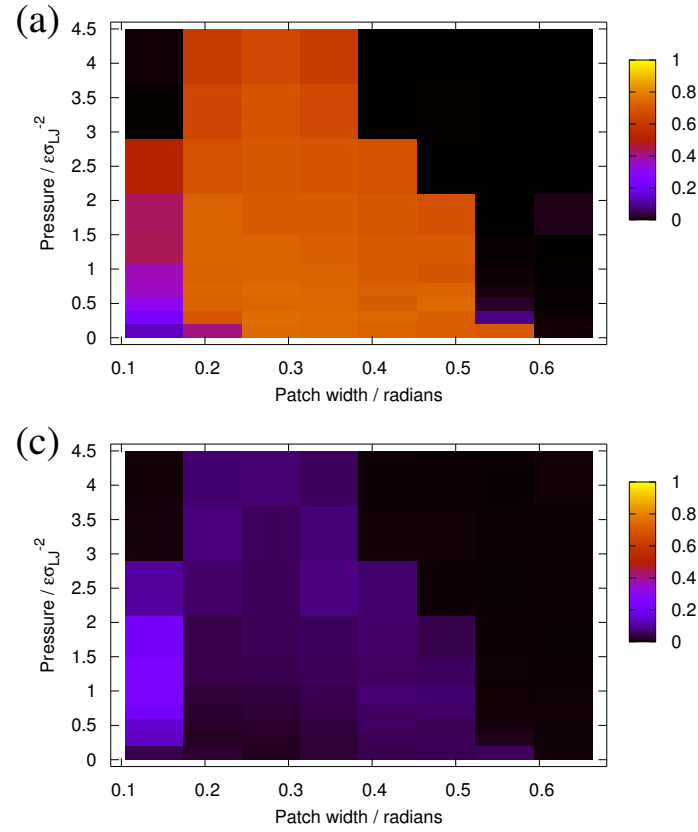

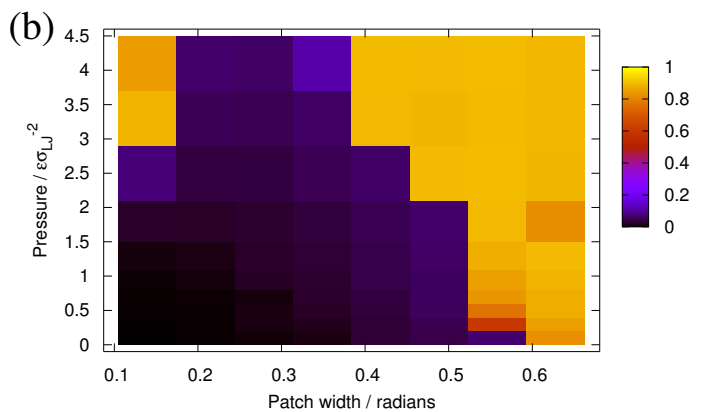

(d)

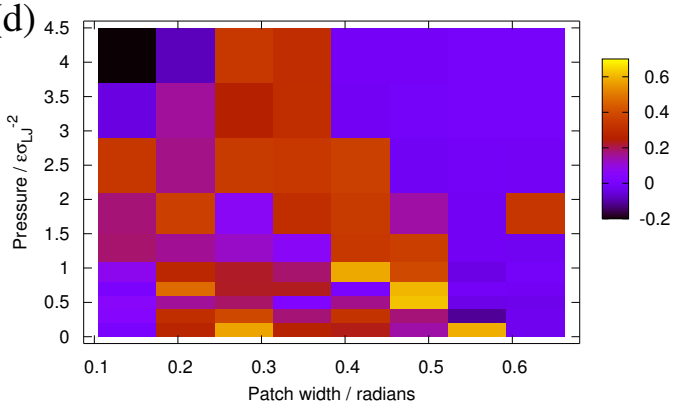

FIG. 8. Dependence on pressure and patch width of the fraction of particles in (a) $\sigma$, (b) $Z$ and (c) $H$ environments, and (d) $|F(\nu=12)|-|F(\nu=6)|$, a measure of the twelvefold character of the diffraction pattern, for the final configuration after annealing.

boundary in Fig. 2 because of the entropic stabilization of the hexagonal phase mentioned earlier.

As one moves away from this boundary, the number of hexagonal atoms slowly decreases towards zero and the degree of twelvefoldness generally drops. Examining the configurations in Fig. 9 one sees a gradual crossover from the quasicrystalline configurations with hexagonal atoms at the centre of the characteristic dodecagons to a pure $\sigma$ phase in Fig. 9(b). For example, about a half of the domains in Fig. 9(d) are pure $\sigma$ and the rest are quasicrystal-like. This crossover is driven by the hexagonal environments becoming energetically increasingly unfavourable as the patches become narrower.

At the narrowest patch width we considered, the lowenergy crystals are the distorted versions of the $\sigma$ and $H$ crystals in which three of the patches point directly at neighbouring particles (Sect. III A 1). These two crystal forms are nearly degenerate, and the structure that results from annealing is a mixture of the two. Even in their distorted forms these two crystals can form coherent boundaries between them, and alternating series of layers of the two crystal forms can be seen in Fig. 9(a).

It is also noticeable from Fig. 8 that for higher pressures at the narrowest patch width hexagonal crystals again form. This change reflects the energetic destabilization of the $\sigma$ phase at these patch widths because the particles can no longer form five strong interactions.

\section{B. 7-patch particles}

Like the 5-patch particles, the 7-patch particles have a local symmetry that is incompatible with crystalline order. However, in addition it is also physically unfeasible for a particle to have seven neighbouring atoms at the equilibrium pair separation without particles overlapping. The question is then how do the particles manage to maximize their patch-patch interactions? When the patches are reasonably narrow, the solution the system finds is to use only five of the seven patches, and to adopt the same types of square-triangle tilings as for the 5-patch system. Figure 10 shows the arrangement of the particles in the $\sigma$ and $H$ environments, and it can be again seen that the $\sigma$ environment has a smaller average deviation of the patch vectors from the interparticle vectors.

Because of this tendency to form square-triangle tilings, the behaviour of the 7-patch system is very similar to the 5-patch system, and so we will much more briefly review the behaviour of this system. The zero temperature phase diagram shows a very similar form with a hexagonal crystal having lowest enthalpy at high pressure and patch width, and a $\sigma$ crystal at lower pressures and patch widths. The crossover between these forms occurs at slightly narrower patch widths than for the 5patch system, because the 7 patches makes the potential somewhat closer to the isotropic limit. As for the 5-patch system at very narrow patch widths, this structure distorts so that three of the seven patches point directly at their neighbours, but this time the acute angle in the hexagonal units is $51.43^{\circ}$ not $72^{\circ}$. 
(a)

(b)
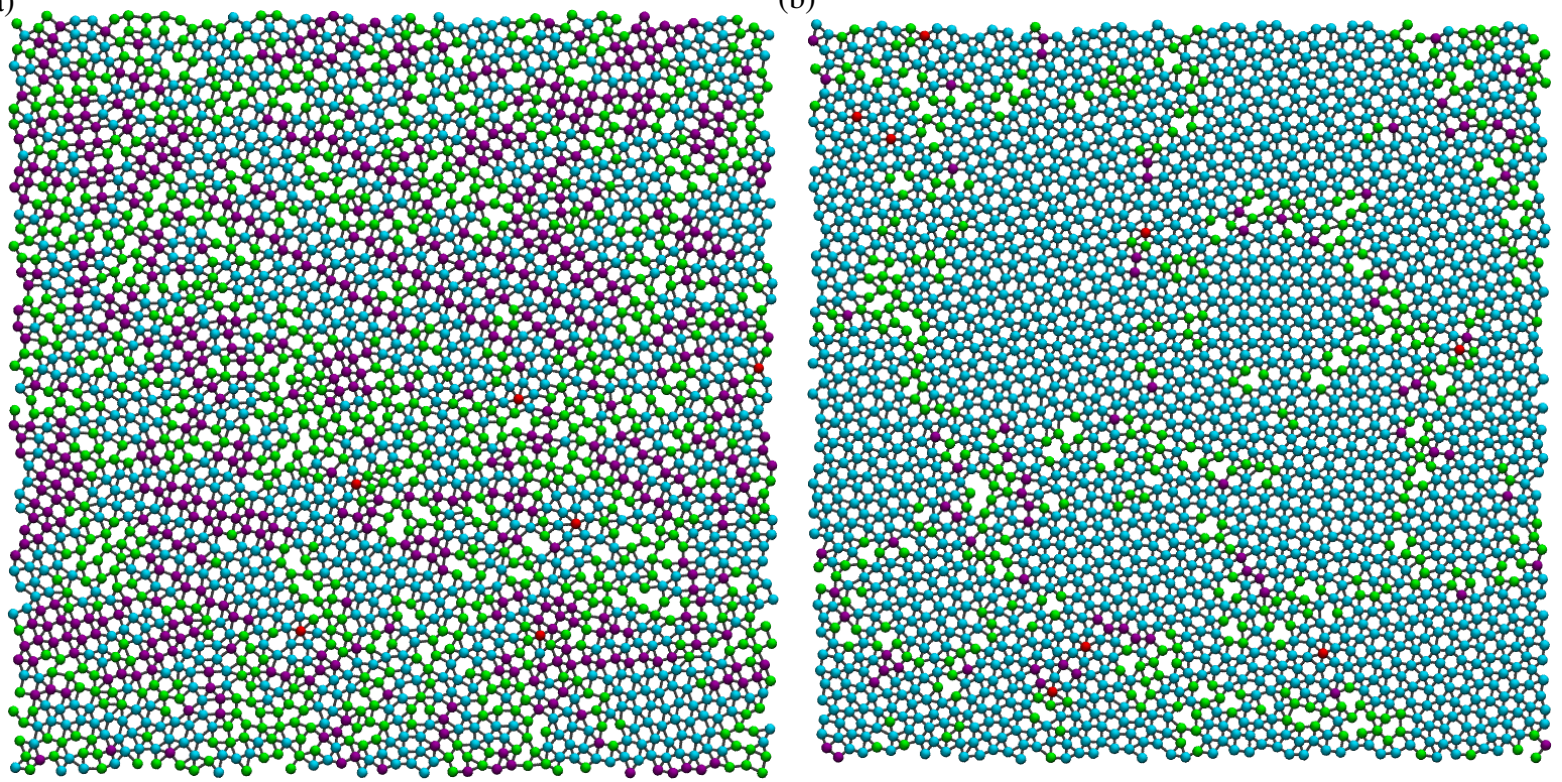

(c)

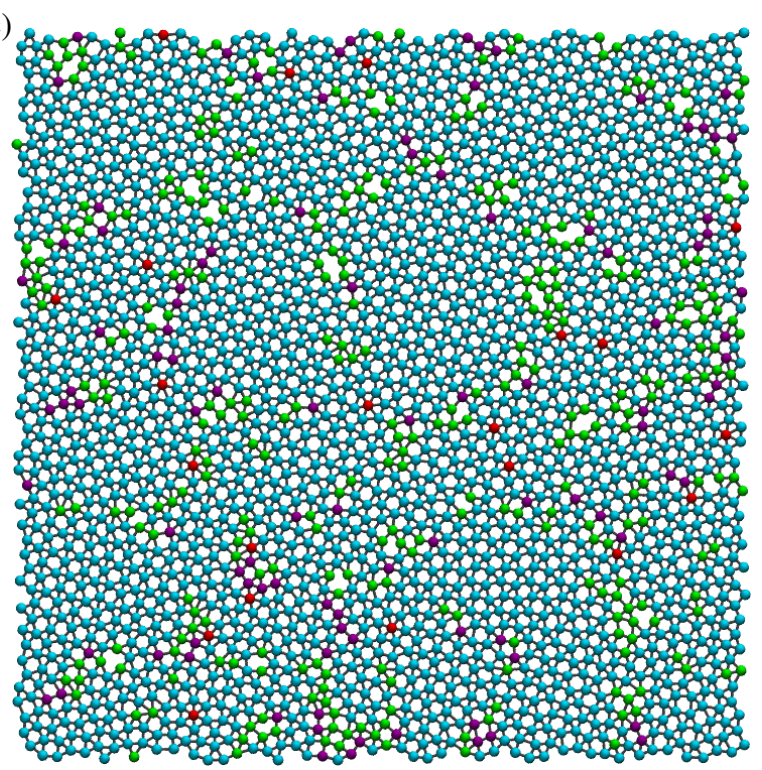

(d)

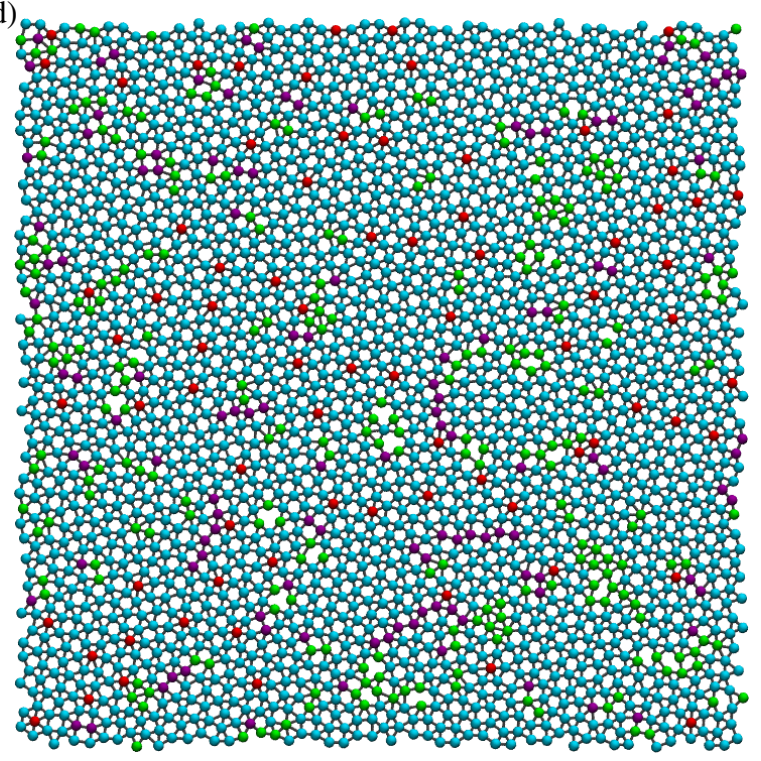

(e)

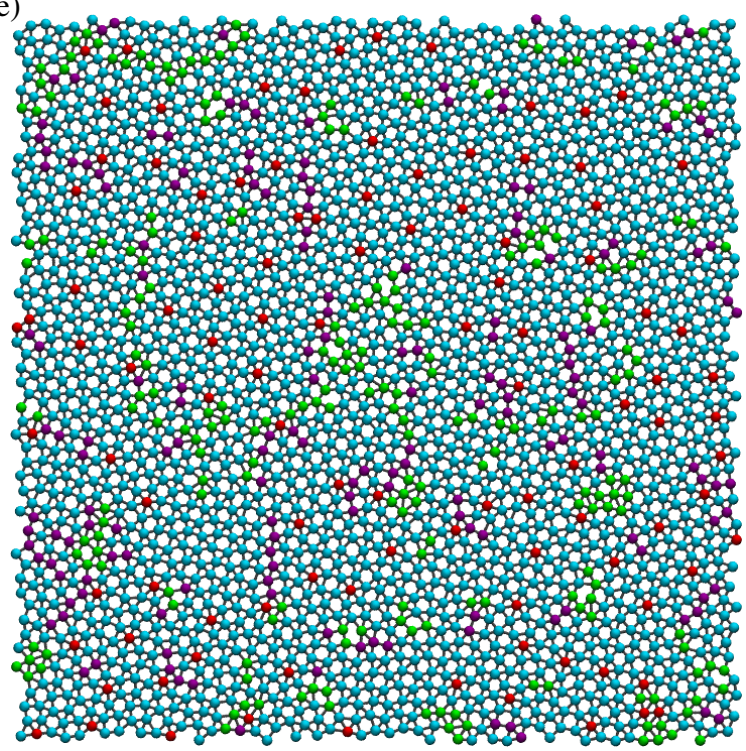

(f)

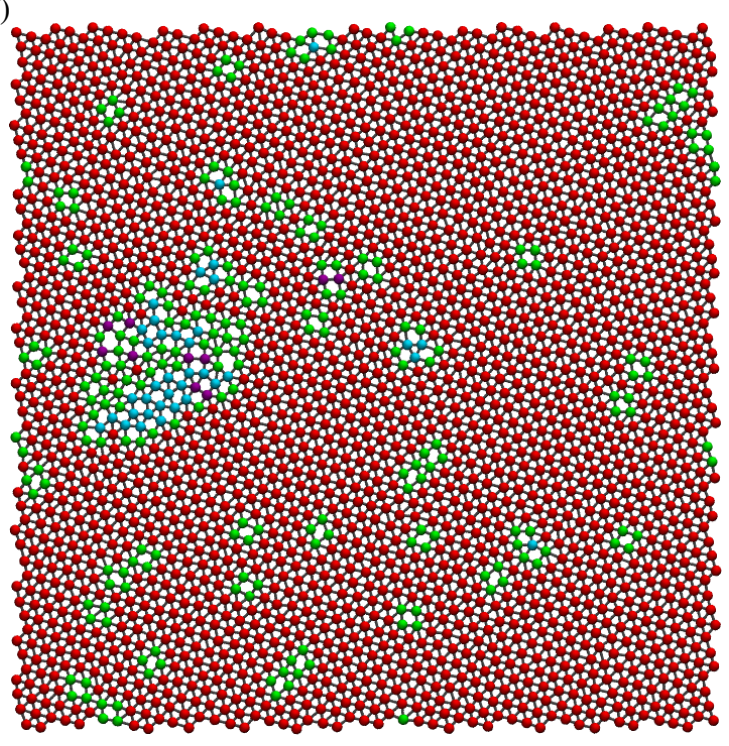

FIG. 9. Dependence of the final configuration after annealing on patch width (a) $\sigma_{\mathrm{pw}}=0.14$, (b) $\sigma_{\mathrm{pw}}=0.21,(\mathrm{c}) \sigma_{\mathrm{pw}}=0.28$, (d) $\sigma_{\mathrm{pw}}=0.35$, (e) $\sigma_{\mathrm{pw}}=0.42$ and (f) $\sigma_{\mathrm{pw}}=0.56$. All are at $p=0.7 \epsilon \sigma_{\mathrm{LJ}}^{-2}$. 
(a)

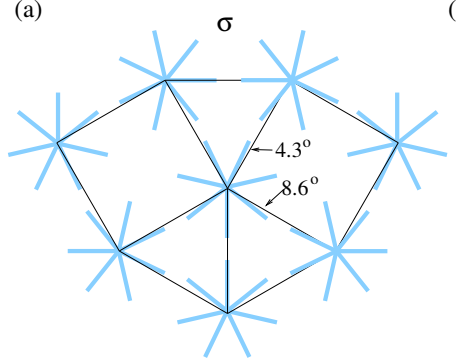

(b)

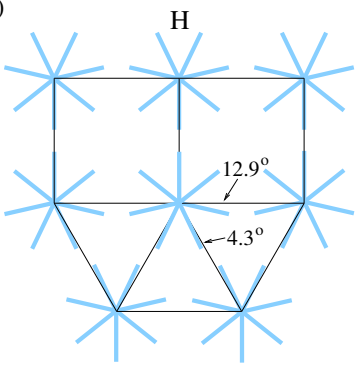

FIG. 10. Deviations of the patch vectors from the interparticle vectors in the (a) $\sigma$ - and (b) $H$-type environments for 7-patch particles.

Again close to the hexagonal $/ \sigma$ boundary our annealing simulations result in quasicrystalline configurations with clear 12-fold diffraction patterns (Fig. 11). Inspection of the configurations also again shows the same dodecagonal motifs (Fig. 7) as found for the 5-patch system.

\section{CONCLUSIONS}

Here we have presented results for a simple twodimensional patchy particle system that exhibits a rich ordering behaviour, in particular showing the formation of dodecagonal quasicrystals based on square-triangle tilings for certain parameter ranges for particles with five and seven regularly arranged patches. These examples can be added to the increasing list of model systems that have been shown to form quasicrystals in simulations 54 The system also provides a nice model system that illustrates how the local structural propensities can lead to complex globally-ordered structures. In particular, quasicrystals were observed in the region of parameter space near to where the enthalpically preferred structure changes from five-fold to six-fold coordination, because of the presence of both coordination environments in the quasicrystal. Interestingly, the structure of the quasicrystals is quite similar to model squaretriangle dodecagonal quasicrystals produced by inflation rules $[58[66,68$

One interesting question concerning the quasicrystals that we observe is whether they are thermodynamically stable or just a kinetic product. As the enthalpy difference between the quasicrystal and the crystal is not that large, it is not unreasonable that the quasicrystal could become thermodynamically stable due to the entropy associated with the many possible configurations for the quasicrystal. However, to confirm this hypothesis would require the free energy of the quasicrystal (or the free energy difference between it and the crystal) to be computed, but it is not clear how this could be achieved. During some of the cooling simulations, the system passes from a liquid to a hexagonal crystal and then to a quasicrystal as the temperature is decreased, showing that there is a parameter range where the quasicrystal is more

stable than the hexagonal crystal. However, on heating the $\sigma$ crystal, we have never seen it transform into a quasicrystal, but instead it directly melts.

An interesting contrast to the behaviour we see here is provided by particles that have five-fold symmetry in their repulsive interactions, $\frac{70 / 71}{2}$ rather than their attractions. Although hard pentagons show an interesting set of solid phases structures, ${ }^{70}$ none of them are quasicrystalline.

Another important question is whether a system that shows behaviour similar to our model could be experimentally realized. Methods to synthesize patchy colloids and nanoparticles are developing rapidly, but as far as we are aware, there are none yet available that could produce, say, particles with a five-fold symmetric distribution of patches. By contrast, effectively restricting such colloidal systems to two-dimensions would be relatively straightforward. For example, if there is a density mismatch between the colloids and the solvent, sedimentation can lead to monolayer formation at the base of the sample, as has been done in recent experiments where a two-dimensional Kagome lattice was assembled from 'triblock Janus' particles ${ }^{33}$

Another possible system in which these dodecagonal quasicrystals could be potentially realized is the multiarm DNA motifs produced by the group of Chengde Mao 72 [9] Each arm is made of two-parallel double helices and both have dangling single-stranded ends that allow them to bind to other such motifs with a well-defined relative orientation. These effective torsional constraints on the interactions lead to quasi-two-dimensional growth be it into sheets ${ }^{72}$ or closed shells ${ }^{77 / 78}$ Interestingly, similar to the equivalent patchy particles, the 3-, 4- and 6-arm motifs form two-dimensional honeycomb, ${ }^{[72 / 73}$ square ${ }^{74 / 75}$ and hexagona ${ }^{76}$ lattices. Furthermore, the five-arm motifs can form a $\sigma$-phase like lattice ${ }^{77}$

However, there are also a number of significant differences between the DNA multi-arm motifs and patchy particle systems. Firstly, the central loop to which the stiff double helices are connected has a certain degree of flexibility allowing the relative angles of the arms to vary. By contrast, the positions of the patches in our model are fixed.

Secondly, the "valence" of the DNA motifs is fixed; i.e. the five-arm motifs can only ever bond to five other such motifs. By contrast, for our patchy particles at intermediate values of the patch width, the particles can adopt five-fold or six-fold coordination environments; it is this flexibility that allows the system to form the quasicrystals. Therefore, if the DNA motifs are to be able to form a quasicrystal, a mixture of 5- and 6-arm motifs with the right stoichiometry would be required. For an ideal dodecagonal quasicrystal, the required ratio of 5and 6 -arm tiles would be $12.928: 1$. However, even with such a mixture, it might be that the system prefers to phase separate into $\sigma$ and hexagonal crystals, similar to what has been seen for mixtures of 3- and 4-arm tiles ${ }^{79}$

In future work, we tend to explore this possibility 

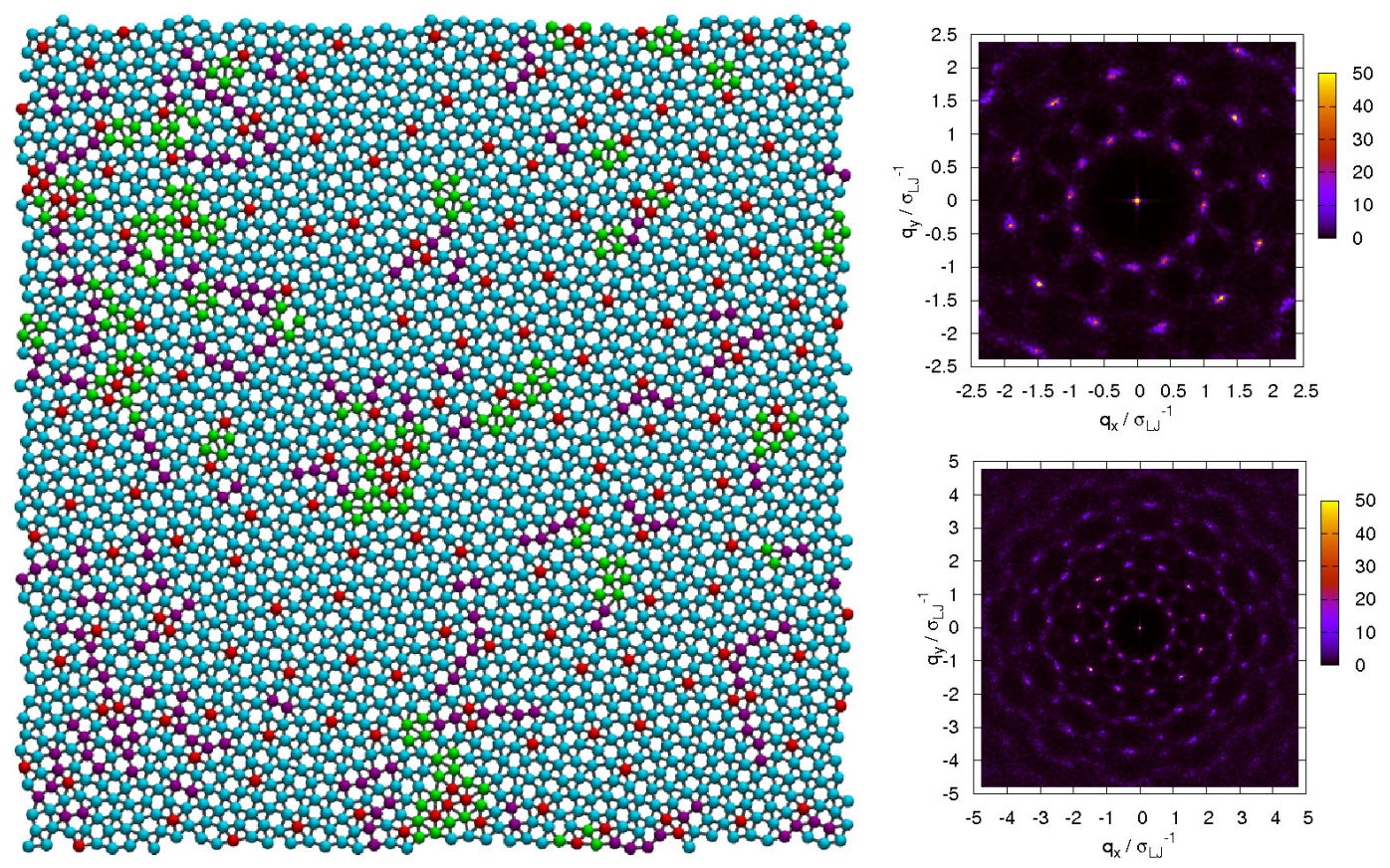

FIG. 11. (a) Configuration after annealing and (b) and (c) associated diffraction patterns (for two different ranges of $\mathbf{q}$ ) at $p=0.7 \epsilon \sigma_{\mathrm{LJ}}^{-2}, \sigma_{\mathrm{pw}}=0.42$ for a system of 7 -patch particles.

further, first by using a coarse-grained model of DNA that we have recently developed 80181 to characterize these DNA multi-arm motifs, including their flexibility and the angular specificity of their interactions. Secondly, this information will then be used to create a patchy-particle representation, where the patch positions are not rigidly fixed but are constrained by an internal potential.

\section{ACKNOWLEDGMENTS}

The authors are grateful for financial support from the EPSRC and the Royal Society.

${ }^{1}$ D. Shechtman, I. Blech, D. Gratias, and J. W. Cahn, Phys. Rev. Lett. 53, 1951 (1984).

${ }^{2}$ X. Zeng, Curr. Opin. Colloid In. 9, 384 (2005).

${ }^{3}$ X. Zeng, G. Ungar, Y. Liu, V. Percec, A. E. Dulcey, and J. K. Hobbs, Nature 428, 157 (2004).

${ }^{4}$ V. Percec, M. R. Imam, M. Peterca, D. A. Wilson, R. Graf, H. W. Spiess, V. S. K. Balagurusamy, and P. A. Heiney, J. Am. Chem. Soc. 131, 7662 (2009).

${ }^{5}$ K. Hayashida, T. Dotera, A. Takano, and Y. Matsushita, Phys. Rev. Lett. 98, 195502 (2007).

${ }^{6}$ S. Fischer, A. Exner, K. Zielske, J. Perlich, S. Deloudi, W. Steurer, P. Lindner, and S. Förster, Proc. Natl. Acad. Sci. USA 108, 1810 (2011).

${ }^{7}$ D. V. Talapin, E. V. Shevchenko, M. I. Bodnarchuk, X. Ye, J. Chen, and C. B. Murray, Nature 461, 964 (2009).

${ }^{8}$ B. Chen, X. Zeng, U. Baumeister, G. Ungar, and C. Tschierske, Science 307, 96 (2005).

${ }^{9}$ A. Takano, W. Kawashima, A. Noro, N. Isono, N. Tanaka, T. Dotera, and Y. Matsushita, J. Poly. Sci. B 43, 2427 (2005).

${ }^{10}$ E. V. Shevchenko, D. V. Talapin, N. A. Kotov, S. O'Brien, and

C. B. Murray, Nature 439, 55 (2006).
${ }^{11}$ Y. Matsushita, K. Hayashida, and A. Takano, Macromol. Rapid Commun. 31, 1579 (2010).

${ }^{12}$ S. Lee, M. J. Bluemle, and F. S. Bates, Science 330, 349 (2010).

${ }^{13}$ X. Zeng, R. Kieffer, B. Glettner, C. Nürnberger, F. Liu, K. Pelz, M. Prehm, U. Baumeister, H. Hahn, H. Lang, G. A. Gehring, C. H. M. Weber, J. K. Hobbs, C. Tschierske, and G. Ungar, Science 331, 1302 (2011).

${ }^{14}$ J. Mikhael, J. Roth, L. Helden, and C. Bechinger, Nature 454, 501 (2008).

${ }^{15}$ J. Mikhael, M. Schmiedeberg, S. Rausch, J. Roth, H. Stark, and C. Bechinger, Proc. Natl. Acad. Sci. USA 107, 7214 (2010).

${ }^{16} \mathrm{~J}$. Mikhael, G. Gera, T. Bohlein, and C. Bechinger, Soft Matter 7, 1352 (2011).

${ }^{17}$ P. Bartlett, R. H. Ottewill, and P. N. Pusey, Phys. Rev. Lett. 68, 3801 (1992).

${ }^{18}$ M. D. Eldridge, P. A. Madden, and D. Frenkel, Nature 365, 35 (1993).

${ }^{19}$ M. D. Eldridge, P. A. Madden, and D. Frenkel, Mol. Phys. 79, 105 (1993).

${ }^{20}$ A.-P. Hynninen, C. G. Christova, R. van Roij, A. van Blaaderen, and M. Dijkstra, Phys. Rev. Lett. 96, 138308 (2006).

${ }^{21}$ A.-P. Hynninen, J. H. J. Thijssen, E. C. M. Vermolen, M. Dijkstra, and A. van Blaaderen, Nature Materials 6, 202 (2007).

${ }^{22}$ S. C. Glotzer and M. Solomon, Nature Materials 6, 557 (2007).

${ }^{23}$ V. N. Manoharan, M. T. Elsesser, and D. J. Pine, Science 301, 483 (2003).

${ }^{24}$ Y.-S. Cho, G.-R. Yi, J.-M. Lim, S.-H. Kim, V. N. Manoharan, D. J. Pine, and S.-M. Yang, J. Am. Chem. Soc. 127, 15968 (2005).

${ }^{25}$ Y.-S. Cho, G.-R. Yi, S.-H. Kim, S.-J. Jeon, M. T. Elsesser, H. K. Yu, S.-M. Yang, and D. J. Pine, Chem. Mater. 19, 3183 (2007).

${ }^{26}$ A. B. Pawer and I. Kretzschmar, Langmuir 24, 355 (2008).

${ }^{27}$ A. Perro, E. Duguet, O. Lambert, J.-C. Taveau, E. BourgeatLami, and S. Ravaine, Angew. Chem. Int. Ed. 121, 367 (2009).

${ }^{28}$ D. J. Kraft, J. Groenewold, and W. K. Kegel, Soft Matter 5, 3823 (2009).

${ }^{29}$ A. Perro and V. N. Manoharan, Angew. Chem. Int. Ed. 26, 18669 (2010). 
${ }^{30}$ S. Jiang, Q. Chen, M. Tripathy, E. Luijten, K. S. Schweizer, and S. Granick, Adv. Mater. 22, 1060 (2010).

${ }^{31}$ D. J. Kraft, J. Hilhorst, M. A. P. Heinen, B. Luigjes, and W. K. Kegel, J. Phys. Chem. B 115, 7175 (2011).

${ }^{32}$ E. Duguet, A. Désert, A. Perro, , and S. Ravaine, Chem. Soc. Rev. 40, 941 (2011).

${ }^{33}$ Q. Chen, S. C. Bae, and S. Granick, Nature 469, 381 (2011).

${ }^{34}$ Z. Zhang, A. S. Keys, T. Chen, and S. C. Glotzer, Langmuir 21, 11547 (2006)

${ }^{35}$ J. P. K. Doye, A. A. Louis, I.-C. Lin, L. R. Allen, E. G. Noya, A. W. Wilber, H. C. Kok, and R. Lyus, Phys. Chem. Chem. Phys. 9, 2197 (2007).

${ }^{36}$ E. G. Noya, C. Vega, J. P. K. Doye, and A. A. Louis, J. Chem. Phys. 127, 054501 (2007).

${ }^{37}$ E. G. Noya, C. Vega, J. P. K. Doye, and A. A. Louis, J. Chem. Phys. 132, 234511 (2010).

${ }^{38}$ F. Romano, E. Sanz, and F. Sciortino, J. Phys. Chem. B 113, 15133 (2009).

${ }^{39}$ F. Romano, E. Sanz, and F. Sciortino, J. Chem. Phys. 134, 174502 (2011).

${ }^{40}$ G. Doppelbauer, E. Bianchi, and G. Kahl, J. Phys.: Condens. Matter 22, 104105 (2010).

${ }^{41}$ E. Bianchi, R. Blaak, and C. N. Likos, Phys. Chem. Chem. Phys. 13, 6397 (2011).

${ }^{42}$ F. Romano and F. Sciortino, Soft Matter 7, 5799 (2011).

${ }^{43}$ M. Antlanger, G. Doppelbauer, and G. Kahl, J. Phys.: Condens. Matter 23, 404206 (2011).

${ }^{44}$ M. Widom, K. J. Strandburg, and R. H. Swendsen, Phys. Rev. Lett. 58, 706 (1987).

${ }^{45}$ P. W. Leung, C. L. Henley, and G. V. Chester, Phys. Rev. B 39, 446 (1989).

${ }^{46}$ E. A. Jagla, Phys. Rev. E 58, 1478 (1998).

${ }^{47}$ A. Skibinsky, S. V. Buldyrev, A. Scala, S. Havlin, and H. E. Stanley, Phys. Rev. E 60, 2664 (1999).

${ }^{48}$ M. Dzugutov, Phys. Rev. Lett. 70, 2924 (1993).

${ }^{49}$ A. Quandt and M. P. Teter, Phys. Rev. B 59, 8586 (1999).

${ }^{50}$ A. S. Keys and S. C. Glotzer, Phys. Rev. Lett. 99, 235503 (2007).

${ }^{51}$ M. Engel and H.-R. Trebin, Phys. Rev. Lett. 98, 225505 (2007).

${ }^{52}$ M. Engel, M. Umezaki, H.-R. Trebin, and T. Odagaki, Phys. Rev. B 82, 134205 (2010).

${ }^{53}$ C. R. Iacovella, A. S. Keys, and S. C. Glotzer, (arxiv:1102.5589).

${ }^{54}$ A. Haji-Akbari, M. Engel, A. S. Keys, X. Zheng, R. G. Petschek, P. Palffy-Muhoray, and S. C. Glotzer, Nature 462, 773 (2009).

${ }^{55}$ A. Haji-Akbari, M. Engel, and S. C. Glotzer, Phys. Rev. Lett. 107, 215702 (2011).

${ }^{56}$ J. C. Johnston, N. Kastelowitz, and V. Molinero, J. Chem. Phys. 133, 154516 (2010).

${ }^{57}$ J. C. Johnston, N. Kastelowitz, and V. Molinero, J. Phys. Chem. Lett. 2, 384 (2011)

${ }^{58}$ M. Oxborrow and C. L. Henley, Phys. Rev. B 48, 6966 (1993).

${ }^{59}$ A. W. Wilber, J. P. K. Doye, A. A. Louis, E. G. Noya, M. A. Miller, and P. Wong, J. Chem. Phys. 127, 085106 (2007).

${ }^{60}$ A. W. Wilber, J. P. K. Doye, and A. A. Louis, J. Chem. Phys. 131, 175101 (2009).

${ }^{61}$ A. J. Williamson, A. W. Wilber, J. P. K. Doye, and A. A. Louis, Soft Matter 7, 3423 (2011).

${ }^{62}$ F. C. Frank and J. S. Kasper, Acta Crystallogr. 11, 184 (1958).

${ }^{63}$ F. C. Frank and J. S. Kasper, Acta Crystallogr. 12, 483 (1959).

${ }^{64}$ D. P. Shoemaker and C. B. Shoemaker, in Introduction to Quasicrystals, edited by M. V. Jaric (Academic Press, London, 1988) pp. $1-57$.

${ }^{65}$ B. Grünbaum and G. C. Shephard, Tilings and Patterns (W. H.
Freeman, New York, 1987).

${ }^{66}$ P. Stampfli, Helv. Phys. Acta 59, 1260 (1986).

${ }^{67}$ J. Hermission, C. Richard, and M. Baake, J. Phys. I. 7, 1003 (1997).

${ }^{68}$ X. Zeng and G. Ungar, Philos. Mag. 86, 1093 (2006).

${ }^{69}$ M. O'Keeffe and M. M. J. Treacy, Acta Cryst. A 66, 5 (2010).

${ }^{70}$ T. Schilling, S. Pronk, B. Mulder, and D. Frenkel, Phys. Rev. E 71, 036138 (2005).

${ }^{71}$ K. Zhao and T. G. Mason, Phys. Rev. Lett. 103, 208302 (2009).

${ }^{72}$ Y. He, Y. Chen, H. Liu, A. E. Ribbe, and C. Mao, J. Am. Chem. Soc. 127, 12202 (2005).

${ }^{73}$ Y. He and C. Mao, Chem. Commun. , 968 (2006).

${ }^{74}$ H. Yan, S. H. Park, G. Finkelstein, J. H. Reif, and T. H. LaBean, Science 301, 1882 (2003).

${ }^{75}$ Y. He, Y. Tian, Y. Chen, Z. Deng, A. E. Ribbe, and C. Mao, Angew. Chem. Int. Ed. 44, 6694 (2005).

${ }^{76}$ Y. He, Y. Tian, A. E. Ribbe, and C. Mao, J. Am. Chem. Soc. 128, 15978 (2006)

${ }^{77}$ C. Zhang, M. Su, Y. He, X. Zhao, P.-A. Fang, A. E. Ribbe, W. Jiang, and C. Mao, Proc. Natl. Acad. Sci. USA 105, 10665 (2008).

${ }^{78}$ Y. He, T. Ye, C. Zhang, A. E. Ribbe, W. Jiang, and C. Mao, Nature 452, 198 (2008).

${ }^{79}$ Y. He, Y. Tian, Y. Chen, A. E. Ribbe, and C. Mao, Chem. Commun., 165 (2007).

${ }^{80}$ T. E. Ouldridge, A. A. Louis, and J. P. K. Doye, Phys. Rev. Lett 104, 178101 (2010).

${ }^{81}$ T. E. Ouldridge, A. A. Louis, and J. P. K. Doye, J. Chem. Phys. 134, 085101 (2011).

\section{SUPPLEMENTARY INFORMATION}

\section{A. Model quasicrystal}

As a comparison to the diffraction patterns we have calculated from our simulated configurations, here we report the diffraction pattern from a model dodecagonal quasicrystal. Dodecagonal tilings can be created by projection from a four-dimensional space but usually include rhombs as well as squares and triangles. An alternative approach to create dodecagonal square-triangle tilings is to use an inflation approach where an increasingly large structure is iteratively built up by applying rules for scaling simpler motifs (in our case squares and triangles) and then replacing them by more complex ones. ${ }^{[5166}[68]$ Here we use what has been termed the "extended Schlottmann" inflation rules! $67 / 68$ The effect of these rules is at each iteration to replace each vertex in the square-triangle tiling by one of the dodecagons in Fig. 7 (a) and (b) depending upon the local geometry of the vertex. Supplementary Figure 1(a) shows the structure generated after 3 iterations of these rules starting from an initial centred hexagon. The corresponding diffraction pattern has clear 12-fold symmetry (Supplementary Fig. 1(b)) and a very similar pattern of peaks as those in Fig. 5 and 11 

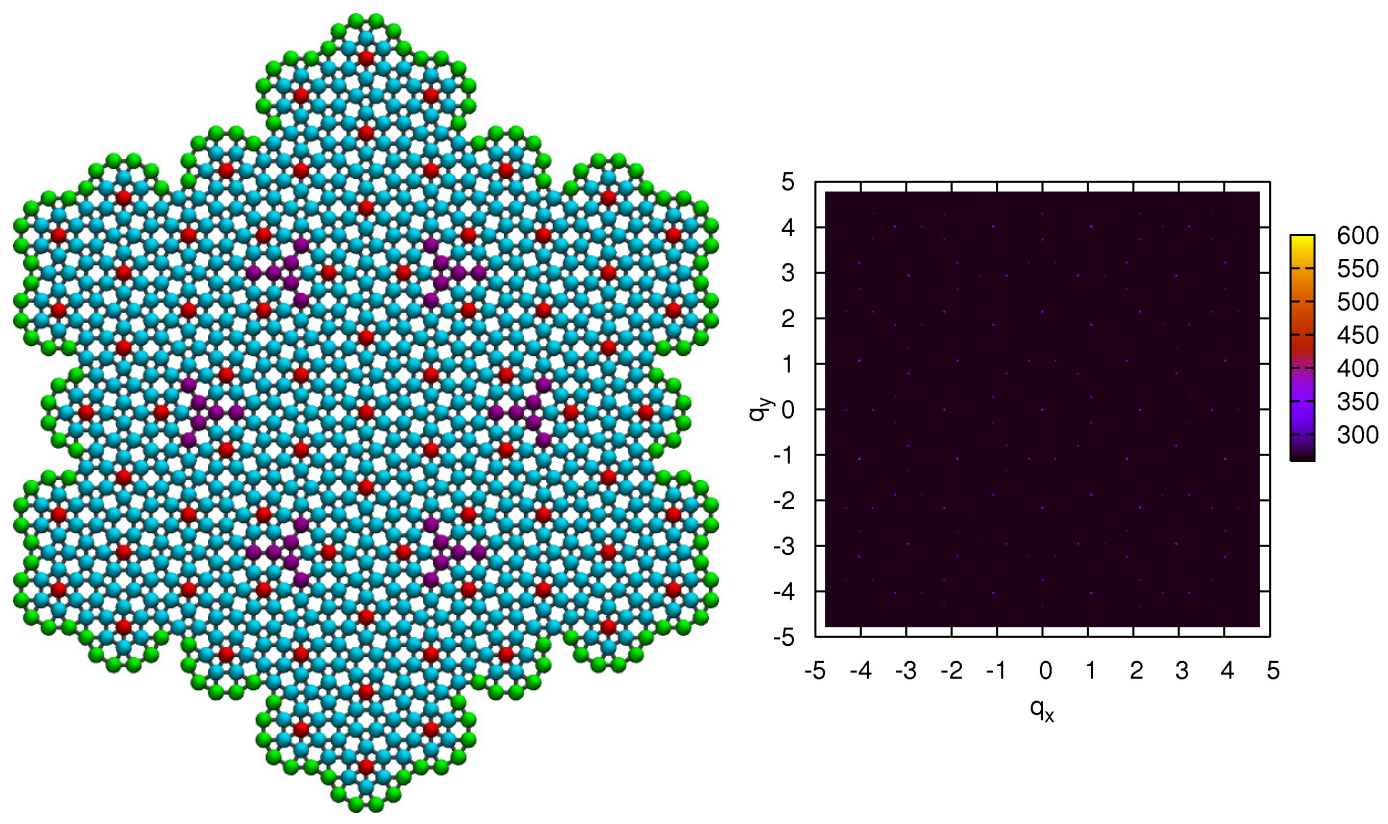

FIG. 1. (a) 1063-particle model quasicrystal generated by three applications of the extended Schlottmann inflation rules 5 and (b) the associated diffraction pattern. The resolution we used in (b) was $\Delta q_{x}=\Delta q_{y}=0.05 \sigma_{\mathrm{LJ}}^{-1}$. 